\title{
I-131 uptake in the breast from fat necrosis
}

\author{
Joseph T. Hoang, BS ${ }^{a}$ (D), Matthew R. Weissenborn, MD ${ }^{b}$ (D), Joseph J. Spigel, MD ${ }^{b}$, Brian J. Welch, MD ${ }^{c}$ (D) and \\ Stanley J. Grossman, MD \\ ${ }^{a}$ Department of Medicine, Texas A\&M College of Medicine, Dallas, Texas; ${ }^{b}$ Department of Radiology, Baylor University Medical Center \\ at Dallas, Dallas, Texas; 'Department of Internal Medicine, Baylor University Medical Center at Dallas, Dallas, Texas
}

\begin{abstract}
Radioactive iodine I-131 whole-body scintigraphy is used to monitor thyroid cancer metastases after total thyroidectomy. We present a case of a woman who was diagnosed with papillary thyroid cancer and underwent a total thyroidectomy. I-131 scintigraphy revealed abnormal accumulation of radioactive iodine in the right breast causing concern for thyroid cancer metastasis. Mammographic studies confirmed that the abnormal radiotracer accumulation was due to fat necrosis in the breast. I-131 uptake in fat necrosis is a poorly understood process that is not clearly defined in the literature. This case highlights that false-positive uptake of I-131 can mimic metastases of thyroid carcinoma.
\end{abstract}

KEYWORDS Breast; fat necrosis; I-131; thyroid cancer

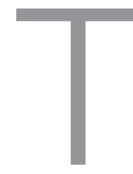

hyroid cancer is the most common endocrine cancer worldwide. ${ }^{1,2}$ Differentiated thyroid cancers (papillary and follicular) constitute up to $90 \%$ of all thyroid neoplasms that originate from follicular cells. $^{3}$ I-131 whole-body scintigraphy is used for surveillance of metastases after total thyroidectomy. Reports of false-positive findings are from physiologic distribution or contamination of radioactive iodine. This case describes an uncommon finding of radioactive iodine uptake on I-131 scintigraphy in the breast due to fat necrosis.

\section{CASE DESCRIPTION}

A 27-year-old woman presented with a palpable mass on the right side of the neck. Ultrasound revealed a $3.6-\mathrm{cm}$ nodule in the right thyroid lobe. Fine needle aspiration demonstrated papillary thyroid carcinoma. She had a total thyroidectomy, and pathological examination confirmed papillary thyroid carcinoma measuring $<1 \mathrm{~cm}$ with extrathyroidal invasion and no lymph node metastases. She was treated with $158 \mathrm{mCi}$ of I-131 radioactive iodide ablation 1 month after surgery. Whole-body scintigraphy 7 days after therapy showed no uptake in the neck, but abnormal radiotracer accumulation was seen within the anterior aspect of the inferior right chest (Figure 1a). A follow-up single- photon emission computed tomography (CT) scan localized the uptake to a $1.6-\mathrm{cm}$ nodular density in the medial right breast (Figures 2a, 2b). An unusual thyroid cancer metastasis was the major concern. Follow-up mammography demonstrated two contiguous fat-containing low-density masses in the right medial and subareolar breast (Figure 2c). Ultrasonography demonstrated mixed echogenicity, predominantly a hyperechoic rimmed $1.2-\mathrm{cm}$ mass with an adjacent subtle mass in the subareolar breast. The combination of findings was favored to represent evolving fat necrosis. One year later, whole-body scintigraphy demonstrated resolution of the I-131 avid lesion in the right breast (Figure 1b). The patient's serum thyroglobulin levels have been undetectable over the past 3 years on followup visits.

\section{DISCUSSION}

The incidence of thyroid cancer increased by $211 \%$ between 1975 and 2013. ${ }^{4}$ This may be due to better means of detection with I-123 scintigraphy and thyroid ultrasound. An estimated 53,990 new cases will be diagnosed this year in the United States, with about three out of four cases affecting women. ${ }^{5}$ Treatment and surveillance of thyroid cancer regularly involve the use of radioiodine isotope I-131. Wholebody scintigraphy detects uptake of I-131, allowing the

Corresponding author: Stanley J. Grossman, MD, Department of Radiology, Baylor University Medical Center at Dallas, 3500 Gaston Avenue, Dallas, TX 75246 (e-mail: stanley.grossman@bswhealth.org)

Color versions of one or more of the figures in this article can be found online at www.tandfonline.com/ubmc.

Received May 30, 2018; Revised August 20, 2018; Accepted August 30, 2018. 


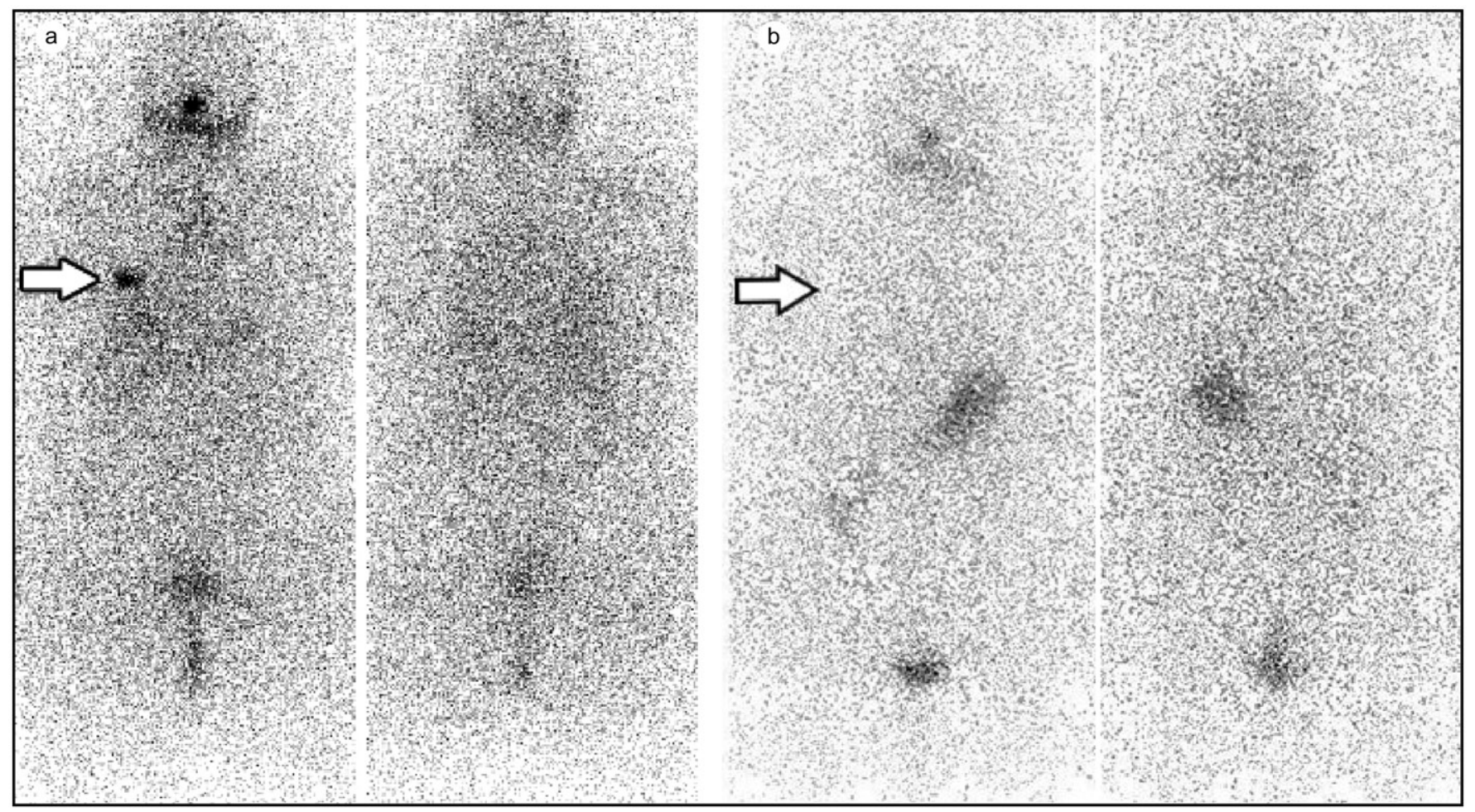

Figure 1. (a) Anterior and posterior planar images from the posttreatment l-131 whole-body scintigraphy show a focus of abnormal tracer localization in the region of the right anterior chest (white arrow). (b) Whole-body scintigraphy 1 year later shows resolution of previous $1-131$ accumulation in the right anterior chest.
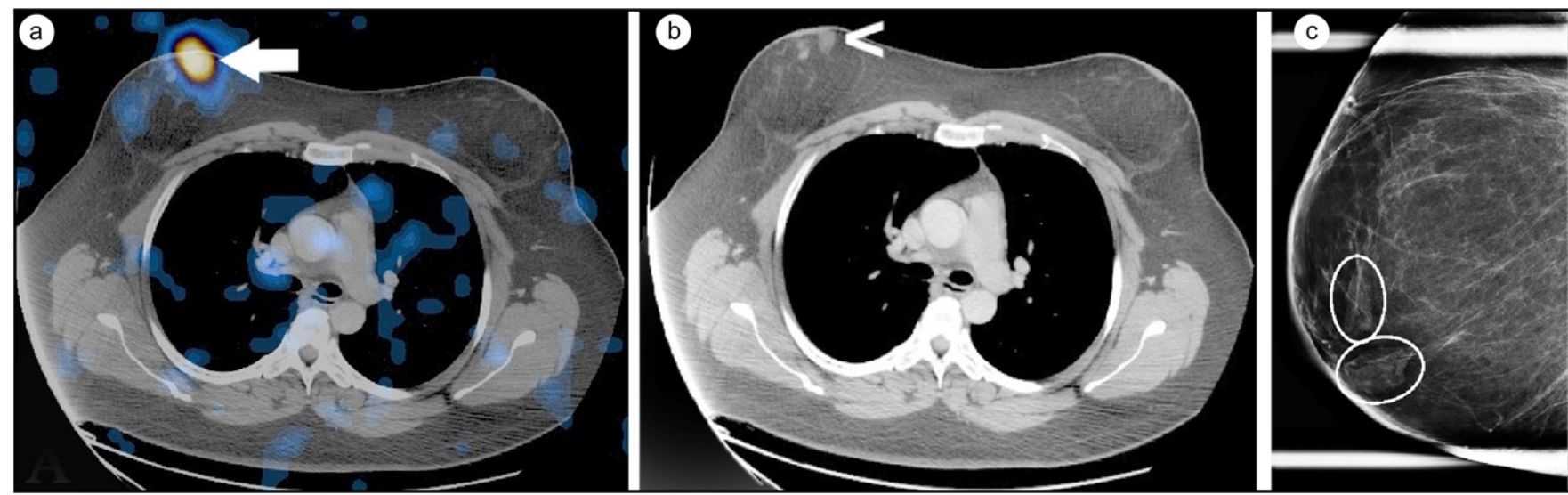

Figure 2. (a) Fused image utilizing axial CT and axial single-photon emission CT images localizes the focus of tracer accumulation to the right breast (solid white arrow). (b) Contrast-enhanced CT image shows two contiguous small soft tissue densities in the right breast (open white arrowhead). (c) Mammographic compression imaging in right mediolateral oblique view reveals two masses of low density in the right medial and subareolar breast containing areas of fat.

visualization and assessment of metastatic or recurrent thyroid carcinoma throughout the body. I-131 uptake into cells depends on the presence of a natrium-iodide symporter, but the full mechanism remains unclear.

Physiologic uptake of I-131 can be seen in tissues outside of the thyroid, including the thymus, salivary glands, lacrimal glands, gastrointestinal tract, liver, and breast. $^{6}$ Radioiodine contamination can be detected in physiologic secretions such as saliva, urine, sweat, feces, and breast milk, causing artifacts unrelated to natrium-iodide symporter function. ${ }^{6,7}$ Inflammatory processes can cause false-positive radioiodine uptake through vasodilation and capillary permeability. Examples of this include hematomas, sinusitis, respiratory infections, myocardial infarction, and cholecystitis. ${ }^{6,7}$ False-positive uptake in the chest region can also be due to thoracic aortic aneurysms or esophageal abnormalities. ${ }^{6}$ Single-photon emission CT can determine whether uptake is intrathoracic or extrathoracic and further localize the lesion to a specific organ or tissue.

Breast uptake of radioiodine is a normal finding in lactating postpartum women due to the increased expression of natrium-iodide symporter in mammary glands to provide sufficient iodine to nursing infants in breast milk. ${ }^{8}$ One study reported that radioiodine uptake occurs in at least $6 \%$ 
of nonbreastfeeding hypothyroid females. ${ }^{9}$ Differential diagnosis of diffuse uptake of radioiodine includes hyperprolactinemia or breast lactation, and focal uptake includes benign cyst, metastatic thyroid cancer, benign tumor, or primary breast malignancy.

Fat necrosis of the breast is a benign inflammatory process that is commonly caused by trauma, breast surgery, radiation therapy, or diagnostic breast interventions. ${ }^{10}$ Fat necrosis appears as an area of hemorrhage in fat that is surrounded by lipid-laden macrophages, histiocytes, and multinucleated giant cells. Fibrosis and calcification from fibroblasts appear in later stages. ${ }^{11,12}$ The mechanism of radioiodine uptake in fat necrosis of the breast is poorly understood. Only one previous case of I-131 uptake in the breast from fat necrosis has been reported in the literature. ${ }^{13}$ The differential for iodine uptake in the chest includes pathologic and inflammatory processes in the lungs, mediastinum, chest wall, and breast. Additional imaging can help determine the true location and etiology for I-131 uptake in the chest.

\section{ORCID}

Joseph T. Hoang (iD) http://orcid.org/0000-0002-2223-3150

Matthew R. Weissenborn (iD http://orcid.org/0000-0003-4878-1187

Brian J. Welch (iD http://orcid.org/0000-0003-2550-5454

1. Siegel RL, Miller KD, Jemal A. Cancer statistics, 2017. CA Cancer J Clin. 2017;67(1):7-30. doi:10.3322/caac.21387.
2. Ferlay J, Soerjomataram I, Dikshit R, et al. Cancer incidence and mortality worldwide: Sources, methods and major patterns in GLOBOCAN 2012. Int J Cancer. 2015;136(5):E359-E386. doi: $10.1002 /$ ijc. 29210.

3. Kitahara CM, Sosa JA. The changing incidence of thyroid cancer. Nat Rev Endocrinol. 2016;12(11):646-653. doi:10.1038/nrendo.2016.110.

4. Lim H, Devesa SS, Sosa JA, Check D, Kitahara CM. Trends in thyroid cancer incidence and mortality in the United States, 1974-2013. JAMA. 2017;317(13):1338. doi:10.1001/jama.2017.2719.

5. American Cancer Society. Cancer Facts \& Figures 2018. Atlanta, GA: American Cancer Society; 2018.

6. Oh J-R, Ahn B-C. False-positive uptake on radioiodine whole-body scintigraphy: Physiologic and pathologic variants unrelated to thyroid cancer. Am J Nucl Med Mol Imaging. 2012;2(3):362-385.

7. Triggiani V, Giagulli VA, Iovino M, et al. False positive diagnosis on ${ }^{131}$ iodine whole-body scintigraphy of differentiated thyroid cancers. Endocrine. 2016;53(3):626-635. doi:10.1007/s12020-015-0750-3.

8. Bakheet SM, Hammami MM. Patterns of radioiodine uptake by the lactating breast. Eur J Nucl Med. 1994;21(7):604-608. doi:10.1007/ bf00285581.

9. Hammami MM, Bakheet SM. Radioiodine breast uptake in nonbreastfeeding women: clinical and scintigraphic characteristics. J Nucl Med. 1996;37(1):26-31.

10. Chala LF, de Barros N, de Camargo Moraes P, et al. Fat necrosis of the breast: mammographic, sonographic, computed tomography, and magnetic resonance imaging findings. Curr Probl Diagn Radiol. 2004; 33(3):106-126. doi:10.1067/j.cpradiol.2004.01.001.

11. Taboada JL, Stephens TW, Krishnamurthy S, Brandt KR, Whitman GJ. The many faces of fat necrosis in the breast. AJR Am J Roentgenol. 2009;192(3):815-825. doi:10.2214/ajr.08.1250.

12. Hogge JP, Robinson RE, Magnant CM, Zuurbier RA. The mammographic spectrum of fat necrosis of the breast. RadioGraphics. 1995; 15(6):1347-1356. doi:10.1148/radiographics.15.6.8577961.

13. Itani M, Lewis DH. I-131 uptake in fat necrosis of the breast. Radiol Case Rep. 2017;12(1):161-167. doi:10.1016/j.radcr.2016.10.018. 